ENCYCLOPEDDIE Encyclopédie berbère

BERBERE

3 | 1986

3 | Ahaggar - Alī ben Ghaniya

\title{
Aire à battre
}

J.-C. Musso et S. Chaker

\section{OpenEdition}

Journals

Édition électronique

URL : http://journals.openedition.org/encyclopedieberbere/2376

DOI : $10.4000 /$ encyclopedieberbere. 2376

ISSN : 2262-7197

\section{Éditeur}

Peeters Publishers

\section{Édition imprimée}

Date de publication : 1 juillet 1986

Pagination : 363-370

ISBN : 2-85744-260-2

ISSN : $1015-7344$

\section{Référence électronique}

J.-C. Musso et S. Chaker, « Aire à battre », Encyclopédie berbère [En ligne], 3 | 1986, document A125, mis en ligne le 01 décembre 2012, consulté le 13 octobre 2020. URL : http://journals.openedition.org/ encyclopedieberbere/2376; DOI : https://doi.org/10.4000/encyclopedieberbere.2376

Ce document a été généré automatiquement le 13 octobre 2020

(C) Tous droits réservés 


\title{
Aire à battre
}

\author{
J.-C. Musso et S. Chaker
}

1 La culture des céréales et en particulier blé et orge pratiquée depuis une très haute antiquité sur tout le territoire maghrébin est régie par tout un ensemble de traditions fortement imprimées dans la mentalité des populations rurales. Culture vivrière capitale pour les habitants, elle exige des opérations de récolte et en particulier la séparation du grain par battage et vannage. Ces deux opérations se pratiquent sur une aire à battre.

2 L'aire à battre s'apelle en kabyle annar pluriel inurar.

Les aires à battre s'aperçoivent de loin grâce a leur surface plane, bien lissée, bordée le plus souvent d'un cercle de pierres. Leur diamètre varie de 6 à $7 \mathrm{~m}$, certaines peuvent atteindre 12 à $13 \mathrm{~m}$ et on en cite même de $20 \mathrm{~m}$ en plaine. En montagne, l'un des côtés est soutenu par un mur en pierres du côté de la pente. Il est fréquent de voir plusieurs aires à battre regroupées les unes auprès des autres pour utiliser au mieux le seul terrain favorable.

3 Généralement, l'aire à battre est une propriété privée, elle appartient à la famille qui a pris soin de l'édifier. Son accès et son utilisation n'en sont pas pour autant exclusifs. Le propriétaire peut en céder, par prêt gratuit, le droit d'usage à ses proches ou à ses voisins. Dans certaines régions, comme l'Aurés ou la Kabylie, l'aire à battre peut être la propriété collective de l'adroum, petit groupe de familles voisines ou alliées qui s'associent pour son exploitation. Exceptionnellement, des aires à battre ont été édifiées par l'ensemble du village. Elles prennent de ce fait la nature de propriété collective et leur utilisation est régie par tour de rôle tiré au sort. 


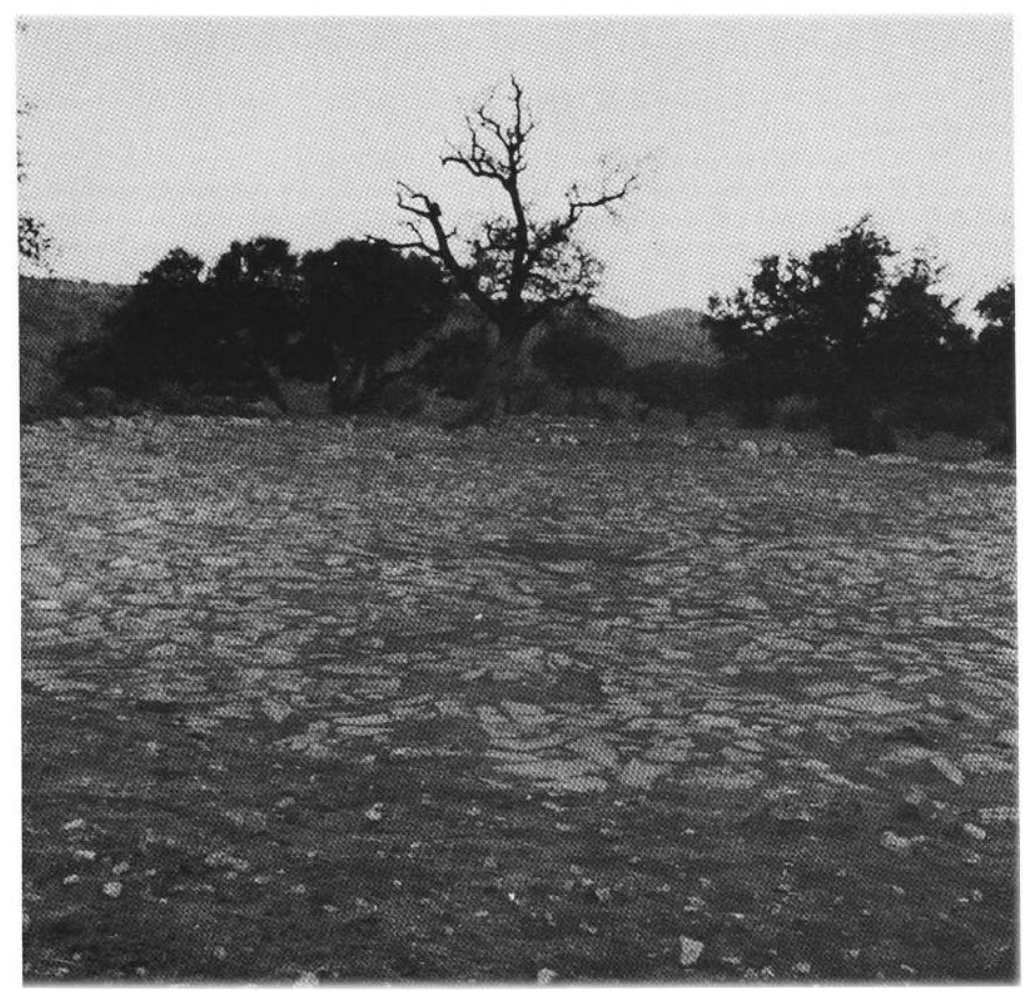

\section{Construction de l'aire à battre}

4 Les modes de construction des aires à battre diffèrent peu sur tout le territoire de l'Afrique du Nord; construction qui est conditionnée avant tout par la nature et la configuration du terrain. Elle sera plus aisée en région de plaine qu'en région montagneuse.

5 Certaines exigences sont impératives : trouver un terrain aussi plat que possible ; rester à proximité des champs des cultures, sans être trop loin de la maison et des greniers à paille, orienter l'aire pour profiter au mieux des vents dominants.

6 Le propriétaire choisit, autant que possible sur son propre terrain l'emplacement nécessaire, et l'aire sera alors édifiée sur un bien melk en propriété propre. Mais quelquefois, surtout dans les régions montagneuses où les emplacements favorables sont plus rares, les conditions nécessaires peuvent ne pas être trouvées sur son propre bien ; l'agriculteur s'adresse alors à la communauté et obtient facilement l'autorisation d'édifier son aire personnelle sur un bien commun, mechmel; cette autorisation est accordée sous réserve qu'une certaine quantité de grain soit versée au profit de la communauté villageoise. Mais sous cette condition, l'aire à battre est propriété de celui qui l'a édifiée et il en use prioritairement selon ses besoins, mais seulement durant l'année en cours.

7 Ce terrain doit être le plus proche possible de la récolte sur pied.

8 L'orientation de l'aire est capitale ; lors du vannage, le vent fera le gros du travail en emportant la balle et en laissant retomber sur l'aire le grain soulevé par la pelle du vanneur. 
9 Les aires à battre sont exposées au vent dominant à cette époque de l'année. Au Maroc, se sont les vents d'ouest, mais dans le Maghreb central et en particulier en Kabylie, se sont les vents d'est, secs et chauds. Si possible, l'aire à battre est édifiée au nord et tout près d'un ou de quelques arbres, afin de profiter de leur ombre pendant les heures les plus chaudes de la journée de travail.

10 Le terrain ayant été choisi en tenant compte de toutes ces conditions, il reste à envisager la construction de l'aire. Avant toute mise en œuvre des techniques comme avant toute construction, il est indispensable de se concilier les forces magiques qui interviennent dans toutes les actions humaines et qui peuvent être favorables mais aussi très nuisibles. Il faut donc chasser les esprits mauvais et attirer au contraire les esprits bénéfiques. Avant tout travail, le groupe familial ou seulement le maître des champs et de la récolte va semer du sel sur la terre choisie comme emplacement. Ce sel a pour effet de disperser les Djnoun (pluriel de Djinn), génies malfaisants.

Puis on procède à un sacrifice sanglant propitiatoire. On choisit un chevreau ou un jeune bouc, de préférence noir, qui est égorgé sur l'emplacement même. Le jeune animal ainsi sacrifié porte en Kabylie le nom de aderri bunnar, c'est-à-dire: la protection, la couverture de l'aire. Le sang est répandu sur la terre en pâture à tous les génies, qui, sans cette offrande ne manqueraient pas de nuire au travail du battage et du vanage.

12 La viande de l'animal sacrifié est consommée en un repas communiel dont les restes sont souvent enterrés dans le sol de la future aire. C'est là encore une offrande aux puissances chthoniennes, comme le repas communiel constitue le lien entre les hommes et les esprits.

13 La peau de la victime sacrifiée, après avoir été tannée, est réservée à la confection du tablier de cuir du moissonneur.

C'est seulement après ces rites propitiatoires que commence la construction de l'aire à battre.

Il est le plus souvent nécessaire de préparer la terre en la piochant assez profondément afin de bien la remuer. Lorsque le terrain n'est pas absolument horizontal, les ouvriers procèdent à son nivellement et doivent construire un mur de souténement du côte de la pente pour en relever le niveau. Ce mur est édifié en pierres souvent rejointoyées avec de la glaise.

Dans les régions méridionales, Aurès, Haut et Anti-Atlas, pour pallier les irrégularités du terrain, les hommes ont coutume de daller la surface utile de l'aire à battre avec de grandes pierres plates, parfois très soigneusement agencées au point de constituer un véritable dallage chez les Chleuh.

17 Enfin les hommes préparent le cercle de pierres qui doit limiter la circonférence de l'aire à battre.

18 Parfois le terrain se présente si favorablement que les hommes n'ont pas à intervenir et que les femmes suffisent à désherber le sol de l'emplacement choisi. C'est ce qui arrive quand on remet en état une aire à battre déjà utilisée les années précédentes.

19 Les femmes apportent ensuite l'eau nécessaire pour mouiller abondamment la terre puis la piétinent longtemps et profondément pour faire une boue bien homogène. Quelquefois, surtout pour les aires de grandes surfaces en particulier dans les régions de plaine, ce piétinement est effectué par des bœufs ou des chevaux. 
20 A la terre bien molle, les femmes ajoutent de la bouse de vache aussi fraiche que possible, de 4 à 5 jours. Il faut compter environ 3 à 4 brouettes de bouse fraiche pour 10 $\mathrm{m}^{2}$ de surface utile.

21 Si la bouse fraiche vient à manquer, on peut utiliser les bouses sèches après leur avoir redonné une consistance pâteuse par malaxage avec de l'eau.

Cet apport de bouse doit être étroitement incorporé à la terre boueuse toujours par piétinement, tâche souvent confiée aux bœufs ou aux chevaux.

23 Après cette opération, on procède au tassement et à l'égalisation de la survace par un damage effectué avec une hie en bois (en kabyle, tamaddazt, pluriel timaddaziri).

Le tassement à la dame et le nivellement terminés il reste à procéder au lissage de la surface qui doit réaliser une croûte assez épaisse et résistante.

25 Selon les régions, après un séchage raisonnable de la couche de boue, les femmes répandent de la paille brisée en l'incorporant à la terre sur une certaine épaisseur, puis après lissage de la surface et séchage naturel, elles balayent l'excès de paille qui n'a pas été incorporé.

Dans d'autres régions, Kabylie en particulier, les femmes incorporent à la surface de la terre des débris de poteries brisées, de la paille et à nouveau de la bouse de vache ; ce mélange est soigneusement tassé et lissé à la main comme un enduit.

27 En Kabylie également, les femmes prennent souvent le soin de lisser la surface de l'aire à battre avec une argile blanche et très fine appelée thoumelalt; argile qui est utilisée très habituellement au crépissage annuel de l'intérieur de la maison. Le lissage de la surface est continué en enduisant les pierres formant margelle sur la circonférence de l'aire. Le soleil, en quelques jours, durcira cette surface lissée et l'aire sera prête à servir.

28 Ce travail long, pénible et surtout minutieux doit être refait tous les ans, à la fin du printemps, pour être prêt à recevoir une nouvelle récolte.

Souvent aussi, en cas de récolte abondante et de travail prolongé sur l'aire à battre, les femmes doivent refaire le lissage détérioré par les sabots des animaux utilisés au dépiquage du grain et cela généralement une fois par semaine.

\section{Les petites aires utilisées par les femmes}

30 En Kabylie, les femmes édifient également sans aucune intervention des hommes des petites aires à battre pour leur usage personnel et exclusif. Ce sont des aires toujours à l'ombre d'un arbre ou d'un mur de la maison, et mesurant $2 \mathrm{~m}$ à 2,50 $\mathrm{m}$ de diamètre. Elles sont quelquefois ovales.

31 Ces petites aires à battre sont utilisées par les femmes pour battre et vanner les petites quantités de céréales, blé ou orge, glanées dans les champs, après la moisson. C'est là également que les femmes trient les pois chiches, les petits pois secs, les lentilles et les fèves de la petite récolte du jardin familial cultivé uniquement par elles.

Ces grains et ces légumes secs sont battus sans l'intervention des animaux, seulement à la main à l'aide d'un battoir en bois appelé azzouz ou également aksar, battoir qui est ordinairement utilisé au lavage de la laine fraîchement tondue. Cet instrument est un tronçon de grosse branche, presque une bûche de 10 à $12 \mathrm{~cm}$ de diamètre et long de 35 à $40 \mathrm{~cm}$ taillé sur une longueur de $15 \mathrm{~cm}$ environ pour aménager une poignée. Il en 
existe aussi de diamètre plus réduit $(6$ à $8 \mathrm{~cm})$ mais beaucoup plus longs $(70$ à $75 \mathrm{~cm})$ muni également d'une poignée taillée dans la masse et d'une surface plane tout le long d'une côté du battoir.

Les graines sont vannées au vent mais à mains nues sans l'aide d'une pelle. Les débris de cosses et les graines ne sont pas projetés en l'air mais seulement élevés par poignées à la hauteur des épaules de la femme assise sur l'aire. Le vent emporte les débris dans leur chute et les graines retombent entre les jambes de l'ouvrière.

\section{Battage et vannage sur l'aire}

L'aire à battre terminée, les travaux de battage peuvent commencer; de façon absolument générale, la séparation du grain de l'épi se fait uniquement par le piétinement des animaux.

Dans les régions montagneuses, comme la Kabylie, les bœufs sont le plus souvent utilisés; les aires sont de surface réduite et une paire de bœufs suffit généralement à mener à terme l'opération. Ils tournent inlassablement sur les gerbes étalées sur l'aire, activés de la voix et du geste par le maître de la récolte.

Mais il est possible de voir également des mulets et plus rarement des chevaux utilisés à ces opérations. Dans certaines régions pauvres, les paysans possédant des récoltes peu abondantes se servent d'ânes pour battre leur grain. Mais dans les régions de plaines, ou sur les hauts plateaux, les aires à battre de plus grande surface accueillent surtout des chevaux, quelquefois quatre de front.

Les animaux sont liés à une même longe dont l'extrémité est attachée à un pieu planté au centre de l'aire. Dans leur déplacement circulaire, partant de l'extérieur, ils enroulent la longe autour du pieu, elle se raccourcit de ce fait et entraîne les animaux à se rapprocher du centre.

Le conducteur les fait alors repartir dans l'autre sens et le déroulement de la longe leur permet de rejoindre le pourtour de l'aire; ils n'ont plus qu'à recommencer le mouvement pour parcourir la totalité de la surface autant de fois qu'il est nécessaire. Le maitre a pour rôle d'activer les animaux par des cris et son fouet; il doit approvisionner les battages en gerbes, il utilise pour cela une fourche en bois à trois dents de fabrication artisanale; avec cette fourche, il veille à la répartition uniforme des épis sous les pieds des animaux.

Après les battages, il reste sur la surface de l'aire la paille brisée, la balle et le grain que l'on sépare par vannage. Le vannage se fait en projetant aussi haut que possible, à un ou même deux mètres au dessus de la tête, paille brisée et grain avec une pelle plate rectangulaire en bois, de fabrication artisanale. Lorsque le mélange est ainsi jeté en l'air d'un mouvement circulaire de la pelle, il s'éparpille et le vent emporte les débris de paille et la balle, le grain plus lourd retombe sur le sol de l'aire à battre.

L'opération se poursuit jusqu'à ce que toute la paille ait été emportée et que seul le grain bien nettoyé reste sur l'aire. Vannage et battage incombent bien sûr à l'homme.

41 La paille brisée et la poussière pénétrent partout dans les vêtements et couvrent les visages des travailleurs, c'est de plus la période la plus chaude de l'année.

Le travail de l'homme est important et dépend de sa volonté mais le travail du vent est indispensable; sans vent, le vannage est impossible. Il faut donc se concilier les forces 
qui commandent aux vents en leur consacrant certaines opérations réputées pour "faire venir le vent». Le bruit fait par le moulin à bras que la femme a sorti de la maison et qu'elle tourne, même à vide, imite le souffle du vent et en provoque la venue par magie sympathique. Les femmes agitent leurs foulards comme si le vent les faisait voler. On suspend dans les arbres voisins des objets que l'on remue comme si leur mouvement était dû au vent. On secoue l'arbre pour que les feuilles s'agitent comme sous l'action du vent. L'imitation du bruit et des mouvements naturels causés par le vent ne peut que provoquer sa manifestation, par magie sympathique.

Pendant que l'homme jette la récolte battue en l'air, la femme rassemble le grain trié sur la partie de l'aire à battre du côté du vent; mais cette opération exige des précautions : il ne faut pas heurter et risquer de blesser les esprits bienveillants qui se tiennent près du grain. Un balai rugueux comme le balai de la maison, généralement fait de bruyère, est trop brutal. En Kabylie, les femmes utilisent un balai spécial, fragile mais beaucoup plus doux, fait souvent en tiges de fougère et aussi en branches feuillues d'asperge sauvage.

\section{L'aire de battage et le sacré}

Pendant toute cette période d'activité, le sol de l'aire est sacré et surtout lorsqu'il a du grain à sa surface, il est interdit d'y pénétrer avec des chaussures, comme dans les lieux saints, mosquées et tombeaux. Les femmes qui ne seraient pas en état de pureté physiologique ne peuvent pénétrer sur l'aire, le sol en est interdit aux tout jeunes enfants qui pourraient involontairement le souiller.

Il serait particulièrement inconvenant de mal se tenir sur l'aire à battre. Il est interdit de siffler, ce qui ferait venir les mauvais génies. On ne doit pas chanter, ni fumer, ni prononcer des mots de mauvais augure tels que : noir, mort, aiguille, charbon, cinq. De plus les hommes qui travaillent sur l'aire sont tenus à une stricte chasteté pendant toute la durée des battages et du vannage.

La récolte est rentrée, les gerbes ont été battues, le grain a été recueilli, l'aire à battre a rempli son rôle et elle entre maintenant en repos, mais sa participation à la vie de tous les jours n'est pas pour autant terminée. Par l'importance de son intervention dans l'existence de la famille et de la communauté agricole, l'aire à battre reste chargée de grande puissance magique. L'aire a ses propres génies « les maitres de l'aire ", elle reste ainsi liée à toutes les forces de fécondité que ce soit celles de la terre ou celles des femmes de la famille.

Même en période de repos, l'aire reste encore un réservoir de baraka et en son enceinte se déroulent de nombreux rites liés naturellement à la fécondité et à la croissance.

Dans les Aurès, le cortège de mariage passant à côté d'une aire à battre s'y arrête, la mariée descend de sa monture et va jeter sur l'aire des dattes sèches. C'est un geste de conciliation vis-à-vis des forces magiques de l'aire ; c'est un rite de fécondité pour la jeune épousée, future mère puisque l'aire contribue à l'abondance des récoltes.

49 En Kabylie, c'est sur l'aire à battre que se déroule le jeu rituel de la kura, jeu de balle disputé entre deux équipes opposées, armées de bâtons en forme de crosse et rappelant le jeu de hockey, jeu rituel pratiqué pour appeler la pluie, gage de fécondité. 
50 Mais en période sèche, si la pluie menace, il suffit d'exposer sur le sol de l'aire à battre un soc de charrue chauffé au rouge pour empêcher la pluie de tomber et de nuire aux récoltes et au séchage des figues.

51 C'est aussi sur l'aire à battre que la qibla, sage-femme traditionnelle, procède à des rites magiques pour empêcher un nouveau-né d'être changé ou possédé par des génies malfaisants. L'eau du premier lavage du nouveau-né est répandue sur l'aire à battre. La qibla utilise aussi l'aire à battre pour pratiquer, secrètement et cachée de tous, un rituel précis destiné à débarrasser la jeune fille de la tusserka, mauvais sort qui frappe certaines filles et les empêche de trouver un époux.

C'est là encore que ces sages femmes pratiquent les sacrifices et rites nécessaires pour conjurer l'action néfaste des génies malfaisants qui empêchent les enfants de marcher et aussi les rites de protection au moment où garçons et filles atteignent leur puberté. Pour cela elle enterre dans le sol de l'aire des mèches de cheveux de ces enfants en même temps que les coquilles des œufs qu'ils ont mangés ensemble.

54 L'aire à battre garde la réputation d'être « Le parquet de Dieu » et les gardiens (a'ssas) esprits bienfaisants mais taquins s'y rassemblent. La terre de son sol est chargée de baraka et jouit d'une grande puissance magique. On en ramasse de petites quantités que l'on place dans un nouet d'étoffe. Ce sachet est suspendu au cou des fiancées pour en assurer la future fécondité.

Les nouets contenant cette terre sont aussi conservés à la maison pour y attirer la baraka de l'aire et préserver les nouveau-nés du mauvais œil.

L'aire à battre, sa construction, la façon dont se déroulent les opérations de séparation du grain et de la paille seraient pour E. Laoust un héritage des Égyptiens conservé par les Berbères. Les pratiques magiques auraient cette même origine.

Le caractère sacré de l'aire à battre et les techniques du vannage au vent existaient déjà chez les grecs. Homère les chante dans l'Iliade $(V, 498)$ « ... le vent emporte la balle sur l'aire sacrée quand on vanne et que la blonde Deme-ter sépare au souffle des vents le grain de la balle ».

On note avec intérêt que la dénomination berbère de l'« aire à battre » est commune à la plus grande partie du domaine. La forme connaît d'ailleurs une morphologie assez particulière au pluriel dans la plupart des dialectes. Ce sont là deux indices de l'ancienneté du terme et de la technique qui lui correspond.

On trouvera chez Laoust (1920, p. 358-359) un relevé très complet des diverses variantes dialectales du vocable. Parmi les plus fréquentes, citons :

- kabyle : annar, pl. inurar (Dallet, p. 574)

- Mzab : anrar, pl. inurar (Delheure, p. 139)

- Chleuh : annrar, pl. innraren (Destaing, p. 12)

- Ghadames : anarar, pl. inarāren (Lanfry, $\mathrm{n}^{\circ}$ 1155)

Le touareg emploie tiyeryert pour désigner l'aire à battre (tiүeryert signifiant ailleurs « surface plane et bien damée ») mais connaît cependant :

-anarar/inûnâr, « enclos solide pouvant se fermer » (Foucauld, III, p. 1394).

Boulifa (1913, p. 390-1) évoque (prudemment) la possibilité d'une étymologie latine (< area ?). En tout état de cause, annar est un terme isolé au plan dérivationnel (de sorte que l'on eut hésité à en dégager la racine : ${ }^{*} N R R,{ }^{*} R R$...) et la morphologie insolite de son pluriel (et ses variations dialectales) laissent supposer une histoire linguistique assez particulière. 


\section{BIBLIOGRAPHIE}

CAMPS G. Monuments et rites funéraires protohistoriques, Paris, A.M.G., 1961, p. 86.

DERMENGHEM E. Le Culte des saints dans l'Islam maghrébin, Paris, Gallimard, 7e édition, 1954, p. 214.

DEVAUX c. Les Kebailes du Djerdjera, Paris, Challamel, 1859, p. 127.

ELIADE M. Traité de l'Histoire des Religions, Paris, Payot, 1974, p. 28.

GAUDRY M. La Femme chaouïa de l'Aurès - Etude de Sociologie berbère, Paris, Geuthner, 1929, pp. 81 $159-160-260-262$.

GENEVOIS H. La Terre - Fichier de Documentation Berbère - Fort National (Algérie) $n^{\circ} 113-1^{\mathrm{er}}$

Trimestre $1972-$ p. $22-23-26$.

hanoteau a. et LetourneuX A. La Kabylie et les Coutumes Kabyles, Paris, Challamel, $2^{e}$ édition, 1893, 3

Tomes, Tome 1 : p. 488 - Tome II : p. 277.

HOMERE. L'Tliade - traduction E. Lasserre, Paris, Garnier, Flammarion, 1965, Chant V vers 498 sq. p. 99.

LAOUST E. Mots et Choses berbères - Notes de linguistique et d'Ethnographie, Paris, Challamel, 1920, p. $234-390$.

SERVIER J. Les portes de l'année, Paris, Robert Laffont, 1962, p. 218 - 230 sq - $239-245$ - 257 - 259 261.

\section{Annar/Inurar, « aire à battre » (linguistique)}

BOULIFA A. Méthode de langue kabyle, (cours de deuxième année)... Alger (Glossaire, p. 390-1), 1913.

LAOUST E. Mots et choses berbères, Paris, p. 358-9, 1920.

INDEX

Mots-clés : Agriculture, Ethnographie, Linguistique, Magie 